\title{
FORMACIÓN CIUDADANA Y UNIVERSIDAD EN COLOMBIA: POLÍTICAS DE CULTURA Y COMUNICACIÓN PARTICIPATIVA
}

\section{CITIZENSHIP EDUCATION AND UNIVERSITY IN} COLOMBIA: CULTURAL AND COMMUNICATION POLICIES FOR PARTICIPATION

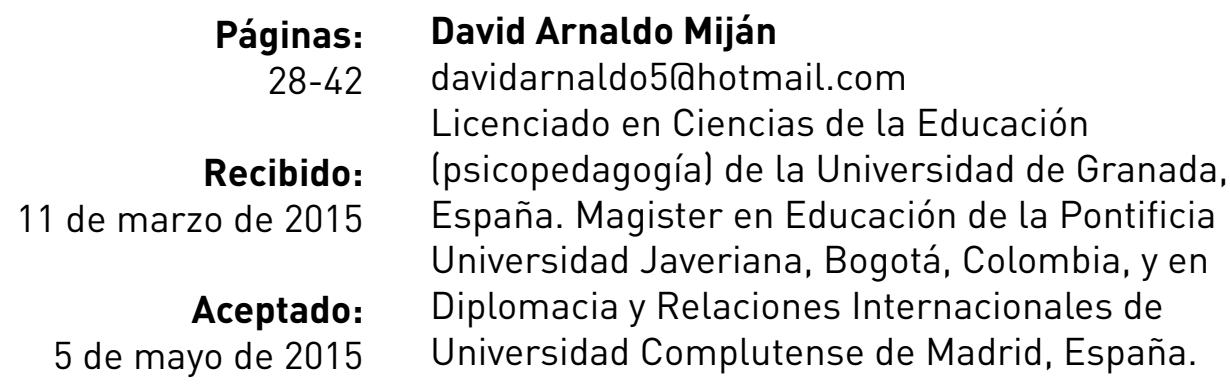

El artículo deriva de la investigación hecha para la tesis de Maestría en Educación titulada: "Formación ciudadana y universidad en Colombia: políticas y ejercicios de cultura participativa". Universidad Javeriana, Bogotá
Resumen

El presente artículo tiene por objetivo describir y analizar, desde la perspectiva de la formación ciudadana y de su relación con una de las cinco dimensiones concernientes a la de las cinco dimensiones concernientes a la función formativa-ética de la Educación $\mathrm{Su}$ perior propuestas por Martínez (2006), cómo perciben actores de dos comunidades univer sitarias colombianas, una de carácter oficial y otra privada, la articulación y efecto de tales
procesos formativos en la cultura participativa e institucional. El análisis de los datos ecogidos a través de la recopilación de información y de entrevistas semiestructuradas se mación y de entrevistas semiestructuradas se de la teoría fundamentada. La muestra fue intencional con cuatro entrevistas utilizando el programa Atlas.ti para mayor rigurosidad. El artículo recopila los resultados, conclusiones y discusiones relativas en el interés de contribuir a la reflexión crítica y el mejoramiento de los procesos de formación ciudadana Educación Superior

Palabras Clave Formación ciudadana, políticas educativas, educación superior, responsabilidad social universitaria, cultura participativa.

\section{Abstract}

This article aims are to describe and analyze from the citizenship education perspective in two Colombian universities, one public and one private, the impacts and effects produce in the universities by the international, national and institutional policy in this matter. Recompilation and analysis were done following the "grounded theory" through four semi-structured interviews chosen intentionally. Atlas ti software was used to process the data. The article presents the results, concitizenship education in both universities.

\section{Keywords}

Citizenship education, educational policy, higher education, social responsibility, participation culture. 


\section{INTRODUCCIÓN}

¿Cómo perciben actores de dos comunidades universitarias colombianas el efecto de los propregunta general, y tomando como referencia el "Ciclo de formulacion de una política pública”, educativa para el caso, que a continuación se reseña, en este apartado se define y delimita el eje central de la investigación de la que da cuenta este artículo.

La pregunta central arriba anotada, se va ajustando a los aspectos más concretos posibles de reconocer en el marco del trabajo. Su delimitación, su acotación, responde a los aspectos problemáticos, conceptuales y prácticos del estudio. En concreto, y respecto al Ciclo de las Políticas Públicas ilustrado en la tabla siguiente, el objetivo de este trabajo se centra en la fase IV, plementacion de la formacion ciudad privada y una oficial) y por to tanto en los asuntos relacionados con la ejecución, gestión y administración, y producción de efectos institucionales.

... La cultura y la política institucional de cada universidad, relativas a las posibilidades de participación de sus estudiantes, profesorado y personal en general, influyen en la formación de este ámbito. Esta influencia repercute en todos los estudiantes, aunque es más notable en aquellos que ejercen el rol de representantes. A pesar de ello, la forma como se organiza el poder en las universidades, el carácter más o menos participativo de los diferentes sectores que la conforman y la transparencia en la toma de decisiones que afectan a su gobierno y funcionamiento, son factores que ayudan a comprender e funcionamiento de las organizaciones. Enseñan, además, en qué medida a participación activa y la implicación en cuestiones que afectan al conjunto de la comunidad son vías válidas para lograr la transformación y mejora. Por el contrario, se convierten en prácticas inútiles si solo

cobran sentido para aquellos que disfrutan construyendo poder a través de la función representativa, sin perjuicio de que ésta haya sido legítimamente adquirida.

M. Martínez Martín (2006), p. 85.

Tabla 1. El Policy Cycle, según Jones (1970).

Fuente: Tomado y adaptado libremente de Meny, Thoening (1992), En Roth Deubel, André-Noël (2003)1.

\begin{tabular}{|c|c|c|c|c|}
\hline FASE I & FASE II & FASE III & FASE IV & FASE V \\
\hline $\begin{array}{l}\text { Identificación de un } \\
\text { problema } \\
\text { Apreciación de los } \\
\text { acontecimientos } \\
\text { Definición de un } \\
\text { problema } \\
\text { Agregado de intereses } \\
\text { Organización de las } \\
\text { demandas } \\
\text { Representación } \\
\text { y acceso de las } \\
\text { autoridades públicas } \\
\begin{array}{c}\text { Demanda de la acción } \\
\text { pública }\end{array}\end{array}$ & $\begin{array}{l}\text { Formulación de } \\
\text { soluciones o acciones } \\
\text { Elaboración de } \\
\text { respuestas } \\
\text { Estudio de soluciones } \\
\text { Adecuación a los } \\
\text { criterios } \\
\text { Propuesta de una } \\
\text { respuesta }\end{array}$ & $\begin{array}{l}\text { Toma de decisión } \\
\text { Creación de una } \\
\text { coalición } \\
\text { Legitimación de la } \\
\text { política elegida } \\
\text { Política efectiva de } \\
\text { acción }\end{array}$ & $\begin{array}{c}\text { Implementación } \\
\text { Ejecución } \\
\text { Gestión y } \\
\text { administración } \\
\text { Producción de efectos } \\
\text { Impacto sobre el } \\
\text { terreno }\end{array}$ & $\begin{array}{c}\text { Evaluación } \\
\text { Reacciones a la acción } \\
\text { Juicio sobre los } \\
\text { efectos } \\
\text { Expresión } \\
\text { Acción política o } \\
\text { reajuste }\end{array}$ \\
\hline
\end{tabular}


Por su importancia, y para Se presentan cinco ámbitos de la formación universitaria en una comprensión más articulada de la fase, se incluyen taciones que favorezcan la formación en valores y el aprenaqui también las intenciona- dizaje ético de los futuros graduados y graduadas: 1) el de los lidades posibles de identificar contenidos curriculares, 2) el de la relación entre estudiantes en la Misión-visión y en los y profesores, 3) el de las formas de organización social de las Proyectos Educativos Univer- tareas de aprendizaje, 4) el de la cultura participativa e instisitarios, a nivel documental, y tucional y 5) el de la implicación comunitaria del aprendizaje en lo que explicitan los conte- académico (p. 85).

nidos de los discursos de actores clave entrevistados.

La hipótesis parte de la re lación ineludible entre universidad y comunidad. Si, se supone, la universidad debe transmitir y comunicar los valores de una ciudadanía responsable a su comunidad y "no puede entenderse una formación universitaria de calidad que no incorpore de forma sistemática y rigurosa situaciones de aprendizaje ético y de formación ciudadana” (Martínez Martín, 2006, p. 85), se quiere apreciar, en primer lugar, si la Misión-visión y el proyecto educativo general de cada universidad incluye principios, fines y valores dirigidos a la formación de ciudadanos y ciudadanas, este será el plano de las intencionalidades.

Luego, para ir al plano de la implementación, se acudirá al análisis de aspectos de la cultura participativa e institucional, partiendo de la propuesta de Martínez Martín (2006).

De estos cincos ámbitos entonces, se toma el cuarto, para lo cual se procedió primero a identificar cómo están organizados los gobiernos universitarios y segundo a contrastar la organización formal con los niveles de participación percibidos por algunos directivos, estudiantes y docentes.

El objeto de este estudio, entonces, se enfoca en particular en a cultura participativa de estas dos universidades. Intenta reconocer cómo la permean las políticas universitarias en que se enmarca. Es un ejercicio que desea, concretamente, ilustrar cómo son los órganos de gobierno de ambas instituciones, qué espacios de representación formal ofrecen a la comunidad universitaria (ejecución y gestión de la política) y cómo son percibidos estos ejercicios por algunos directivos, estudiantes y docentes (percepción de efectos producidos).

Para finalizar solo mecionaré la normativa nacional e internacional en la que se soporta este estudio. En cuanto a las nacionales se procede de las normas más generales, la Constitución Nacional colombiana de 1991, la Ley 30 de 1992 sobre Educación Superior, La Ley 115 de 1994 y el Plan Decenal de Educación 2006 - 2016 y los estatutos de las dos universidades propuestas en el estudio. En la parte internacional se trata aquí en primer lugar las referencias más importantes para conceptualizar este estudio en relación con la significación de la formación ciudadana y espećfficamente en lo tocante a la relación universidad cociedad, para lo cual se retoma el informe “La educación encierra un tesoro" de Jacques Delors (1996) y el informe "Reflexiones y perspectivas de la educación superior en América Latina. Informe final-proyecto Tuning América Latina” (2007). Igualmente nos parece importante en el ámbito más local resaltar el informe de la ASCUN (Asociación Colombina de Universidades) "Politicas y Estrategias para la Educación Superior de Colombia 2006-2010”.

\section{MÉTODO}

Según Ballas, "En metodología cualitativa se trabaja con muestras intencionadas, seleccionadas a partir de un criterio predeterminado por el investigador" (2008, p. 45). Dentro de los muestreos que señala la autora, para este estudio se ha seleccionado el "muestreo de conveniencia” (Ibíd p. 48) como opción que más se ajusta a las condiciones, objetivos y limitaciones del estudio.

\section{Instrumentos}

\section{Tabla 2. Categorías a priori del análisis.}

La selección del instrumento se hizo en función de las necesidades del estudio. De los diferentes tipos de técnicas de recolección de datos se eligió la entrevista individual semiestructurada.

\section{Recolección de datos}

Los datos se han obtenido de dos fuentes diferentes:

- Por un lado se recogieron varios documentos significativos de ambas universidades. (Misión-visión).

- La segunda fuente de información son las entrevistas realizadas en cada universidad.

\section{Trascripción y análisis de datos}

Para la fase de documentos, primeramente se realiza el análisis de su contenido empleando para ello el programa Atlas.ti, con base en las categorías que se definieron.

\section{Categorías a priori}

Intenciones: enunciados referidos a lo que se quiere conseguir en el terreno de la formación de ciudadanos y ciudadanas de acuerdo con sus comprensiones sobre necesidades de convivencia en el país.

Concepciones: cómo se entiende, desde el punto de vista institucional, el hecho de formar ciudadanos

Acciones formativas: acciones concretas que se enuncian y que están en desarrollo o cuyos resultados son ya una realidad.

Participación y representación: enunciados que se refieren a las formas en las cuales los diferentes actores de la universidad forman parte activa de las estructuras y decisiones de las mismas. 
Una vez extraída la información de los cuatro documentos y agrupada con el programa antes mencionado segú las categorías señalas se pasó al análisis de la misma para construir las conclusiones generales de cómo ambas instituciones reflejan en sus documentos oficiales las in tencionalidades, políticas tencionalidades, politicas hacen referencia a sus acciones concretas de formación ciudadana, proceso que se explicará en la última parte de procedimiento.

Para las entrevistas se adopto una forma distinta de procesamiento de la información. En este proceso, igualmente, En este proceso, igualmente,
se empleó el Atlas.ti, basado se empleó el Atlas.ti, basado en la teoría fundamentada permite realizar la codificación de los datos en función de investigación.

Por último, partiendo de esta información, se procedió a elaborar las conclusiones en función de los objetivos del estudio.

\section{RESULTADOS}

A continuación se presentan los resultados obtenidos de la categorización de los documentos consultados y de la codificación hecha en las entrevistas. Estos resultados corresponden y responden únicamente a los datos recogidos y no pretenden ser generalizables sino simplemente ejemplificables. Primero, se hace un análisis descriptivo en función de los objetivos seleccionandos y los enunciados representativos para cada caso. En un andisis posterior, interpretativo, se construyen relaciones entre enunciados por medio de un modelo analítico relacional que responde al objetivo general y específicos y, por último, se elaboran las conclusiones, discusiones y recomendaciones que se consideran pertinentes y relevantes, basadas en los propios aportes de los entrevistados y los propios del investigador.

\section{Análisis documental}

El análisis documental, para el caso de la institución A se basa en el "Proyecto Educativo Institucional, Ser y Naturaleza Universitarios y Misión-visión”. Para la institución B se consultó el documento "Visión y propósitos fundamentales".

De acuerdo con las categorías enunciadas y definidas en el procedimiento y la aplicación de Atlas.ti, se obtuvo la siguiente matriz de contenidos.

Tabla 3. Matriz de enunciados por categorías realizada a partir del análisis documental

$\begin{array}{ccccc}\text { Universidades } & \text { Intenciones y/o } & \text { Concepciones Acciones } & \text { Participación y } \\ \text { Categorías } & \text { Declaraciones } & & \text { formativas } & \text { Representación }\end{array}$

\section{Categorías Declaraciones}

\section{formativas Representación}

A 13

11

11

4

0
Como se puede apreciar en los gráficos siguientes el $90 \%$ de los contenidos en los documentos de ambas universidades hace énfasis en la intenciónes y concepciones que corresponden a las dos primeras categorías y solo un $10 \%$ a aciones de participación concretas.

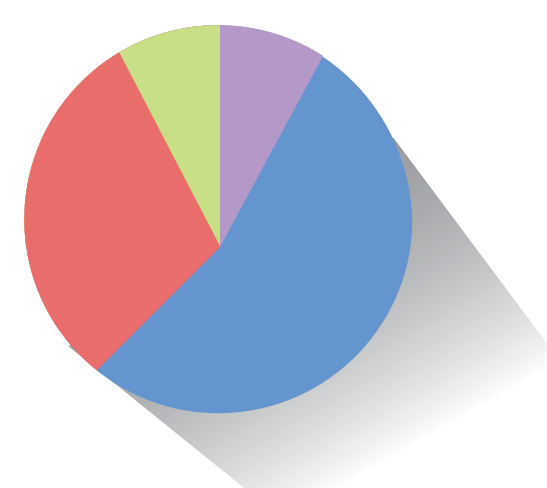

Intenciones Concepciones

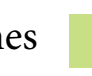

Acciones formativas

Participación

Gráfica 1. Porcentajes categorías universidad A y universidad B.

\section{Categoría intencionalidades}

Ambas universidades tienen una clara intención de formar ciudadanos comprometidos con la sociedad, lo que de por sí ya es satisfactorio. La A (privada) tiene la pretensión de hacerlo a través del servicio y los valores cristianos. La universidad B (oficial) a través del cambio social y la autonomía del individuo.

En la institución privada lo expresan de

la siguiente manera:

Mediante las funciones específicas de toda institución universitaria en búsqueda del saber y abierta a todos los aspectos de la realidad, busca servir a la comunidad humana, en especial a la colombiana, procurando la instauración de una sociedad más civilizada, más culta y más justa, inspirada por los valores que proclama el cristianismo.
Por su parte la institución oficial lo

expresa:

Se trata de hacer explícito un proyecto que restituya el imperio de los principios y valores propios de la nueva institucionalidad y de la sociedad, entre otros: la reafirmación de la nacionalidad; la eticidad y el civismo como guías esenciales de la nueva ciudadanía. 


\section{Categoría concepeciones}

En esta categoría lo que se pretende averiguar es ¿cómo se entiende, desde el punto de vist institucional, el hecho de formar ciudadanos?"

La universidad A así lo escribe:

Por formación integral la universidad Javeriana entiende una modalidad de educación que procura el desarrollo armónico de ción que procura el desarrollo armónico de todas las dimensiones del individuo. Cada
persona es agente de su propia formación. Esta favorece tanto el crecimiento hacia la autonomía del individuo como su ubicación en la sociedad, para que pueda asumir la herencia de las generaciones anteriores y para que sea capaz, ante los desafios y para que sea capaz, ante los desafios de futuro, de tomar decisiones responsables a nivel personal, religioso, científico, cultural y político.

La universidad B lo explica:

- El proyecto político, pedagógico e institucional condensa el conjunto de fines, principios, estrategias políticas, pautas normacipios, estrategias políticas, pautas norma-
tivas, objetivos y procesos educativos que tivas, objetivos y procesos educativos que conducen a la formación del nuevo ciudadano y del modelo de sociedad que consulta las características de nuestra organización histórico-social y del pacto social plasmado

en la Constitución de 199

\section{Categoría de acciones}

\section{formativas}

En ella intentamos identificar las acciones concretas en materia de formación ciudadana con las que cuentan las universidades.

\section{Insitutcion A:}

Apoyar las diferentes asociaciones, movimientos y organizaciones de la comunidad académica, en torno a los propósitos del Proyecto Educativo. Los exalumnos debe ser convocados a una mayor participación en el proceso innovador con miras a que asuman proyectos relacionados con su formación permanente.

Institucion B:

- Convocar a la comunidad universitaria para que se apropie de nuevas formas de asociación, participación, diálogo y concertación y contribuya a la creación de los espacios que hagan posible, de una parte, la construcción y reconstrucción del proyecto de Universidad, y de la otra, el afianzamiento de la cultura, de la tolerancia, paz, la convivencia, la solidaridad, el respaz, la convivencia, la solidaridad, el respeto a los derechos fundamentales y los demás valores científicos, éticos, políticos y cívicos que inspiran a la universidad.

\section{Categoría participación \\ y representación}

Solo la institución oficial tiene un comentario relativo a esta categoría.

- Que el 10 de diciembre de 1991 fueron presentadas a su consideración las propuestas de Misión y Proyecto Educativo de la Universidad A elaboradas, con la participación de la comunidad universitaria, por los Comités Funcionales a quienes Consejo Directivo Universitario con Consejo Directivo Universitario confió esta
tarea.
Efectivamente, los documentos se centran en las intenciones y las concepciones y menos en la duda es si la ausencia es solo formal o es que realmente no hay propuestas o ideas de cómo pasar del discurso a las acciones. En ninguna universidad se encontró otro documento más técnico y aterrizado que concretara como se iba a fomentar la participación, tipo una estrategia concreta de participación.

\section{Análisis entrevistas}

\section{Resultado 1}

Las universidades parten, para los procesos de formación ciudadana, de la intención de desarrollar lo que hoy se conoce como "formación integral". Las declaraciones de la universidad oficial tienen un cariz político y comprometido con la sociedad desde el punto de vista del ejercicio de derechos y deberes ciudadanos. La universidad privada, adicionalmente, relaciona éste compromiso de cumplimiento en derechos y deberes con los valores propios de sus convicciones religiosas. El tránsito entre macropolíticas, políticas nacionales y realidades institucionales está condicionado fuertemente por factores sociopolíticos del contexto nacional y por las tensiones entre participación formal y real. En efecto la primera idea de este trabajo es identificar las intencionalidades de las instituciones en relación con la formación ciudadana que se explicitan en su Misión-visión y Proyecto Educativo Universitario.

\section{Comentarios ejemplificantes:}

- "Hay un PEI, hay un proyecto político pedagógico que salen el año 2000 si no estoy mal, no tengo presente la fecha en este momento, que tiene como precedente la formación política de los estudiantes de la Universidad y su injerencia en la definición no solamente de políticas educativas sino de la política en este país y la formación de pensamiento crítico y ese tipo de cosas, pero a eso se le anteponen otras cosas y el rector de esta universidad ha sido asiduo por ejemplo en criticar el hecho de que aquí no tiene por qué hacerse formación política, aquí por qué tenemos que ver temas relacionados con, no sé el paramilitarismo, o este tipo de cosas aquí no, la Universidad forma maestros no politólogos lo ha dicho..."

- "Yo pienso que si, pues, claro que lo que te decía, hay cosas por mejorar, porque, no se está alcanzando, no se le está dando la magnitud que se le debería dar, o sea para mí, lo que yo te decía, en la misión, en el PEI y todo eso aparece y se está implementando pero yo tengo te decia, en la mision, en el PEI y todo eso aparece y se está implementando pero yo tengo la sensacio

\section{Resultado 2}

Las universidades tienen una clara intención de formar ciudadanos comprometidos con la sociedad: bien a través del servicio y los valores cristianos; bien a través de la promoción del 
cambio social y la autonomía presentatividad. Las decisiones se toman a modo de consenso, individual. donde el rector tiene el poder de decisión más importante, por lo tanto el Consejo asume, para algunas decisiones importantes, Este artículo también inten- más bien un papel consultivo. Existen instancias superiores sin ta describir cómo en los do- representación ni participación de la comunidad universitaria cumentos institucionales y a que orientan las políticas institucionales.

partir de las percepciones de

las personas entrevistadas, es- En la B, el Consejo incluye más actores, aparte de los tres printán organizados los Gobiernos cipales que son comunes. Los procesos de elección son por mucha fuerza y los candidatos siempre son miembros de los mismos. La figura se entiende más como de representación de En cuanto a las percepciones la comunidad correspondiente y no solo como miembro del En cuanto a las percepciones la comunidad correspondiente y no solo como miembro de
de los entrevistados, resu- consejo. Las decisiones son por votación, aunque se evidencian miendo, los gobiernos uni- diferentes niveles, y vinculantes para la universidad, el rector versitarios asumen formas y no tiene la última palabra. No existen instancias superiores de convocan participantes con orientación y decisión.

algunas diferencias: En la A

la comunidad universitaria comentarios ejemplificantes:

está conformada por directi-

vos, profesores y estudiantes, y ahora la inclusión ya aprobada de los egresados; los procesos de elección al Consejo superior son a través de voto indirecto y los candidatos se presentan como independientes. Los elegidos no tes. Los elegidos no son representantes sino miembros del comité, lo que se define como Otro análisis realizado es reconstruir, a partir de las entrevisun papel en el que entra en tas, la percepción de los efectos producidos por la participajuego la democracia participa- ción de los distintos estamentos de la comunidad universitari tiva pero no se traduce en re- en el Consejo superior universitario, sobre la cultura participativa de las universidades.

Tabla. 4. Sistemas de gobierno.

\begin{tabular}{|ccccc|}
\hline Universidad & $\begin{array}{c}\text { Representación } \\
\text { comunidad/ } \\
\text { instancias } \\
\text { superiores de } \\
\text { orientación } \\
\text { Reducida/sí }\end{array}$ & $\begin{array}{c}\text { Procesos de } \\
\text { elección por } \\
\text { votación }\end{array}$ & $\begin{array}{c}\text { Carácter de los } \\
\text { candidatos } \\
\text { independiente } \\
\text { movimientos estu- } \\
\text { diantiles }\end{array}$ & $\begin{array}{c}\text { Función dentro del } \\
\text { consejo } \\
\text { miembro } \\
\text { representante }\end{array}$ \\
A & indirecta & directa & & \\
B & & & \\
\hline
\end{tabular}

que el proyecto educativo, digamos la intencionalisi es muy clara de formar un excelente ciudadano que desde su competencia profesional aporte a la construcción nía, eso es como muy típico de aquí".

\section{Resultado 3}

\section{Comentarios ejemplificantes:}

- "Estamos hasta cierto punto cansados de esa figura, sí, de participación pero que no tiene el mérito de la representación".

- "No pues ¿cómo?, representar, nunca, no, para nada; menos en el Consejo Directivo"

- "las decisiones del Consejo Directivo parten de la idea del consenso, no se hacen por votación, por eso a veces las decisiones son muy complicadas de tomar frente a algunos temas. Casos excepcionales en donde se vean en las personas que están en Consejo Directivo no lleguen a decisiones por consenso la persona que preside el Consejo tiene la potestad para terminar la decisión y decir que va a ser tomada por él”.

\section{Resultado 4}

Los procesos de cultura participativa analizados tienen algunas fortalezas y muchas debilidades aún. Las fragilidades se relacionan con la organización interna, con el débil apoyo que realmente reciben de sus respectivos grupos y con la bajísima participación en los procesos de elección, sobre todo de los estudiantes.

Los movimientos estudiantiles surgen como relevantes en el análisis de la cultura participativa (subcategoría que surge básicamente de la universidad pública). Los movimientos estudiantiles no tienen reconocimiento ni representación formal en los gobiernos universitarios.

La mayoritaria abstención en los procesos de elección, según percepción de alounas personas entrevistadas, parecen mostrar que buena parte de estudiantes y maestros no confían ni en el proceso ni en el papel que sus "representantes" van a tener, lo que contribuye a crear un clima de desaliento y apatía que muy probablemente repercute en su p a sociedad (o viceversa).

Los entrevistados (as) anotan aspectos positivos de los procesos: en particular la organización de las campañas y lo que ellas suponen en cuanto a formación en ciudadanía y participacion tanto a nivel individual como para la comunidad universitaria.

\section{Comentarios ejemplificantes:}

"Es que ese es el problema: es decir, aquí la forma política interna de nuestra organización no es, en un sentido real, democrática y la participación es una participación que no es representativa y si no es representativa, es problemática. Y no se genera una opinión realmente tenga peso".

"Entonces, ahí es claramente la comparación con la realidad: a veces esto no está funcionando y yo creo que sí genera unos imaginarios claros, es: "si ni siquiera funciona en la Universidad, de qué va a servir mi voto por ejemplo después”. Y que yo sí creo que eso es algo que dentro del proceso va en detrimento de alguna manera de la concepción que existe, y más aún cuando ni siquiera es claro el concepto”.

"Que haya una abstención tan alta, que hayan personas que no quieren lanzarse, ya está enviando un mensaje claro y es, acá está pasando algo y esto tiene que servir para algo..."

\section{Resultado 5}

Se trata de constrastar aqui la organización formal con los niveles de participación per- 
cibidos. Se intenta cumplir con la ley, desde la perspectiva formal, pero los procesos de participación tienen aún fragilidades importantes. Las instituciones establecen y promueven formas y canales de participación que se cumplen pero, para la mayoría de los entrevistados (as) aún son insuficientes, y contraproducentes en algunos casos, por su cantidad (porcentajes de participación), forma (información, difusión) $\mathrm{y}$ condiciones.

Se proponen asuntos como mayor equidad en número de representantes, mejorar los procesos de elección y participación, mejorar las condiciones de los procesos en cuanto a información y capacitación de los miembros, más espacios para la vida en comunidad e incluso descargas laborales, compensaciones académicas o armonización ejercer los cargos con mayor responsabilidad.

\section{Comentarios ejemplificantes:}

- "Pero los canales oficiales de hacer comunidad académica no son ni suficientes ni están adecuados para una formación democrática de nuestra época; no pueden ser los mismos que teníamos el siglo pasado, pues por principio y por el cambio constitucional que ha habido en el país, no pueden ser los mismos”.

- "Pero definitivamente, lo primero que tú preguntabas que si ha sido modelo o un ejemplo la dirección de la Universidad: para nada. Nosotros no contamos con ese modelo, o sea, ¿cómo podemos contar con ese modelo, cuando las directivas de la Universidad están casadas con un modelo de universidad, de país, con un modelo político absolutamente sectario? O sea, aquí nuestras directivas aplauden a un gobier- no que a sus enemigos los tilda de terroristas independientemente de quiénes se

"Yo sí creo que debiera haber una instancia profesoral y simultáneamente también una instancia estudiantil, llámese asamblea o no, pero que tuviese de alguna manera voz, participación también y representación”

\section{Conclusiones}

1. Los documentos base de las universidades: Proyecto Educativo Universitario y su Misión y Visión, están adaptados a lo que establecen las políticas y recomendaciones internacionales y nacionales respecto a formación ciudadana; igualmente en lo que establece al respecto el marco legal nacional.

2. Las universidades reflejan en sus órganos de gobierno los compromisos escritos en los documentos.

3. Las intencionalidades que expresan las normas y documentos, en cuanto a formación ciudadana, intentan llevarse a la práctica.

4. A pesar de todo este buen panorama en cuanto a lo legal y a las intencionalidades, la comunidad académica entrevistada entiende que el acercamiento entre discurso y realidad está condicionado tanto por factores del contexto sociopolítico como por las fragilidades de la cultura participativa propia de la relación representados -representantesescenarios de participación que portan tensiones no siempre coherentes con las intencionalidades y normas.

5. Los efectos percibidos en cuanto a la responsabilidad de la universidad de contribuir a formar ciudadanos y ciudadanas son paradójicos: los estudiantes y profesores entienden la universidad como un país en pequeño; algunos de los aprendizajes asociados con las prácticas cotidianas de ejercicio de la ciudadanía son contraproducentes con los esfuerzos de democratización.

\section{Discusión y Recomendaciones}

Se dejan los siguientes puntos como aportes para una discusión más profunda y sobre los que se cree sería interesante investigar:

1. Fortalecer la comunicación entre los diferentes estamentos de la comunidad universitaria y los miembros de los diferentes consejos. Desarrollar la cultura institutcional de la participación.

2. Fortalecer los procesos de elección, dándoles mucha más relevancia, por ejemplo dentro de la asignatura obligatoria de Constitución. Apostar mas decididamente por la formación ciudadana.

3. Que los Consejos y su quehacer sean mucho más conocidos, visibles y comunicados a la comunidad universitaria en general (comunicación participativa).

4. Sería interesante explorar la idea del diseño de una estrategia en participación y comunicación. Detallando en la misma objetivos que se quieren alcanzar, recursos disponibles y actividades concretas.

5. Una demanda muy recurrente y que se dejó clara en las entrevistas es la necesidad de consejos o asambleas de estudiantes o profesores, por ejemplo. Por lo tanto la sugerencia sería que las universidades fomentaran concretamente la creación de estos espacios.

6. Otra discusión podría darse en lo referente a la carga laboral o académica de los representantes-integrantes; así como la mayor representación del número de profesores o estudiantes en los consejos.

7. Una discusión más radical y de mediano plazo sería el estudio, evaluación y reforma profunda del sistema de representación y participación en el pás en general, y en los gobiernos escolares universitarios en particular. 


\section{Referencias}

Ballas Meneses, Claudia Andrea. (2008). Textos de estudiantes. Introducción a los métodos cualitativos. Campos de aplicación en educación y etapas en el proceso de investigación. Bogotá: Universidad Javeriana.

Flick, U. (2004). Introducción a la investigación cualitativa. Madrid: Morata.

Krause, M. (1995). “La investigación cualitativa: un campo de posibilidades”. En Revista temas de Educación, 7, pp. 19-39.

Martínez, Miguel (2006). "Formación para la ciudadanía y Educación Superior”. En Revista Iberoamericana de educación, 42, pp. 85-102.

Roth, André-Noël. (2006). Políticas públicas. Formulación, implementación y evaluación. Bogotá: Ediciones Aurora.

Strauss, A. y Corbin, J. (2002). Bases de la investigación cualitativa. Técnicas y procedimientos para desarrollar la teoría fundamentada. Colombia: Universidad de Antioquia.

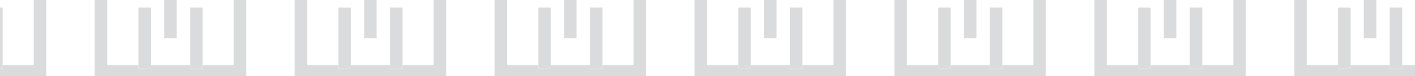

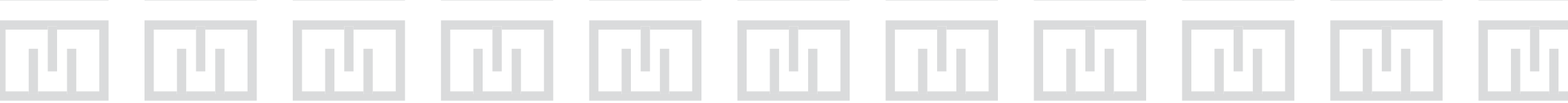

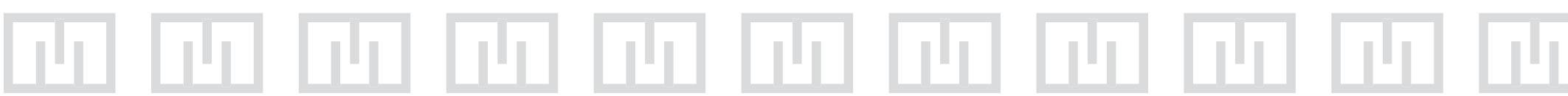

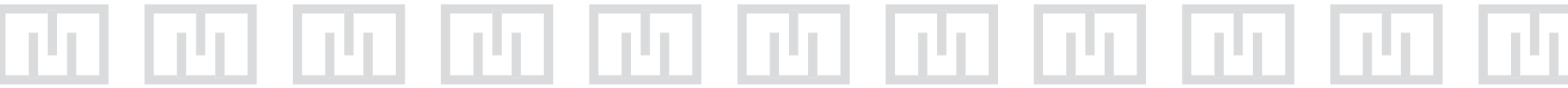

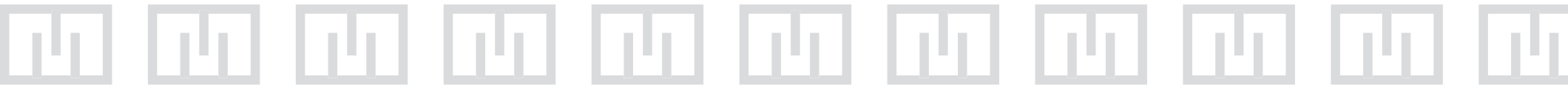

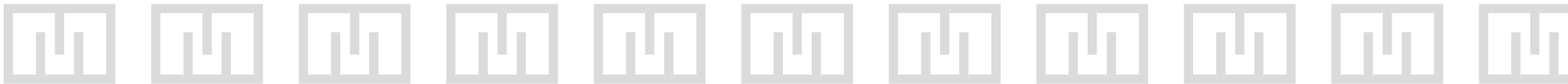

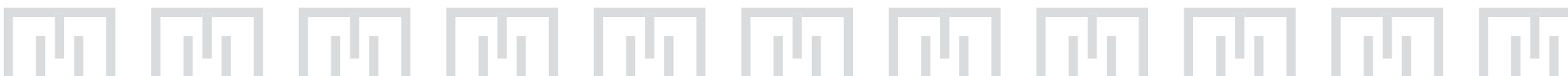

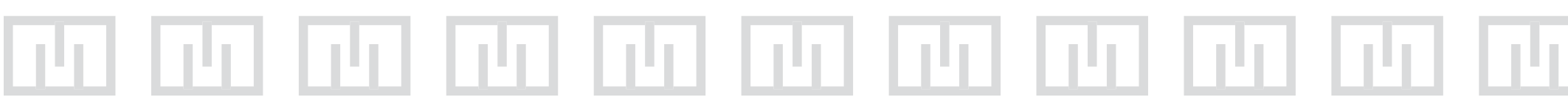

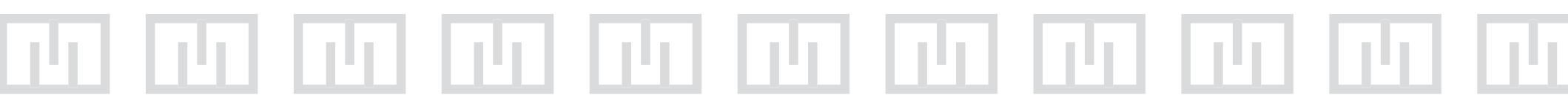

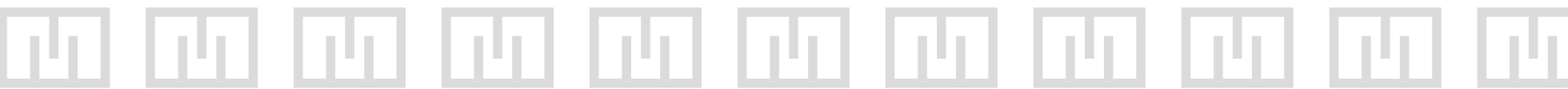

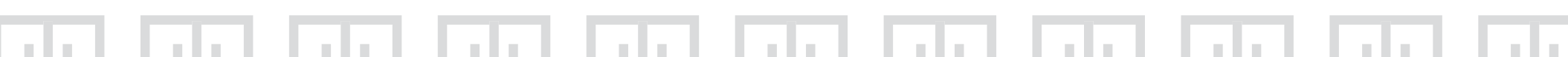
然

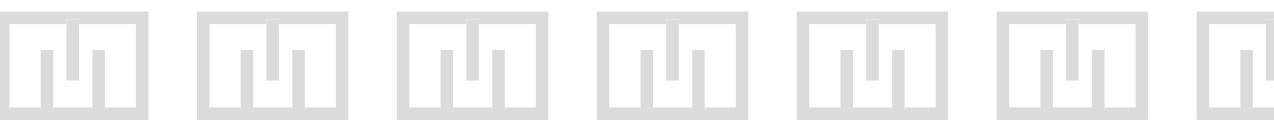
II

II

1 11

1 II 1 |

\section{1} I | 1 II 